\title{
ESTABLECIMIENTO DE PATRONES, UN PILAR FUNDAMENTAL PARA EL JUEGO Y EL APRENDIZAJE
}

Establishment of patterns, a mainstay for play and learning

Estabelecimentos de padrões, um pilar fundamental para o jogo, e o aprendizajem

\section{Desireé López de Maturana Luna}

Junta Nacional de Jardines Infantil, Chile. Fono: 226545000 Correo electrónico:

desireelopezdem@gmail.com

\section{Resumen}

La presente monografía, nos habla sobre patrones o redes de atractores que subyacen a los

juegos libres de niños y niñas y que podrían develar aspectos de su propia epistemología. La investigación que nos permitió identificar aquellos patrones, requirió la observación sistemática de los juegos creados y jugados por niños de 4 y 5 años en un contexto de Jardín Infantil. Estas relaciones se establecen por la forma en que los niños y las niñas se vinculan con el medio y cómo lo aprehenden a través de sus sentidos, absorbiéndolo y transformándose en armonía con el mismo. El tiempo y el espacio es experiencial, inmenso y holístico; caben todos los mundos posibles. Los niños y las niñas van construyendo la experiencia lúdica a partir de una "idea" inicial que surge de la relación circunstancial con el entorno, y luego ésta, va resistiendo todas las transformaciones imaginables según sean las significaciones que cada uno de ellos y ellas le otorguen desde su propia historicidad, a través de una secuencia accional. Así, los juegos se desarrollan como un proyecto que motiva la acción porque se valora el proceso, dado que no existe un objetivo predeterminado. La observación no puede tener un solo foco porque transcurre en una espacialidad multiproxémica, y la sutileza de las interacciones y transformaciones lúdicas, son casi imperceptibles. A estas relaciones que hilvanan aquellos movimientos inesperados que rompen el panorama continuo de espacio y tiempo, los reconoceremos como Patrones o Redes de Atractores.

Palabras clave: juego libre, aprendizaje, patrones, atractores. 


\begin{abstract}
This monograph, talks about patterns or networks underlying attractors free playing of children and could reveal aspects of his own epistemology. The investigation allowed us to identify those patterns required the systematic observation of play created and played by children 4 and 5 years in a context of kindergarten. These relations are established by the way the children are linked with the environment and how it apprehends through his senses, absorbing and transforming in harmony with it. Time and space are immense, experiential and holistic; where all possible worlds fits. Boys and girls are building the playing experience from an initial "idea" that arises from the circumstantial relationship with the environment. Then it goes resisting every conceivable transformations as are the meanings of each one of them, and they will grant from its own historicity, through an actional sequence. Thus, play are played as a project that motivates action because the process is valued, since there is not a predetermined target. The observation may not have a single focus that takes place in a multiproximal spatiality, and subtlety of interactions and playful transformations are almost imperceptible. These relationships that weave those unexpected movements that break the continuous picture of space and time, these are recognize as Patterns or Network's attractors.
\end{abstract}

Key words: free play, learning, patterns, network's attractors.

\title{
Resumo
}

A presente monografía, fala-nos sobre os padrões ou as redes de atractores, que subjacem nos jogos livres das crianças, e que poderia revelar os aspectos da sua própria epistemología. A investigação que permitiu-nos identificar aqueles padrões, precisou da observação sistemática dos jogos criados e interpretados pelas crianças de 4 e 5 anos, no contexto do jardím infantil. Estas relações são estabelecidas pela forma como as crianças estão ligadas absorvendo e transformando em harmornia com o mesmo. O tempo e o espaço, é experiencial, imenso e holítico, onde encaixam todos os mundos possíveis.As crianças constroem a experiência do jogo a partir de uma "ideia" inicial, que surge da relação circunstancial com o meio ambiente, e depois ésta vai a resistir todas as 
transformações possíveis, de acordo aos próprios significados e historicidad, numa sequência de ações. Assim, os jogos desenvolvem-se como um projeto que motiva a ação, porque o processo é validado, e não há um objetivo predeterminado. A observação não pode ter um único foco porque ocorre em uma espacialidade multiproxémica, ea sutileza das interações e transformações lúdicas, são quase imperceptíveis. De esta maneira, essas relações que tecem os movimentos inesperados, que quebram a imagem contínua do espaço e do tempo, nós os reconhecemos como Padrões ou Redes de Atractores.

Palavras-chave: jogo libre, aprendizajem, padrões, atractores.

\section{Introducción}

En Chile estamos viviendo grandes transformaciones en el ámbito político, económico y también social; este contexto ha dado origen a distintas reformas, entre ellas, la educacional que abarca los distintos niveles del sistema. En el marco de este proceso, se le otorgó rango ministerial al nivel parvulario desde la sala cuna, reconociéndolo como primer peldaño o como la puerta de entrada al sistema de educación pública.

Las reformas, resultan por lo general de cambios paradigmáticos que van afectando y tensionando de manera más o menos profunda las estructuras de los sistemas. Sin duda que la reflexión crítica del trabajo educativo en la educación parvularia y los aportes que podemos hacer desde el lugar que nos encontremos, ayuda a la implementación de las políticas públicas de educación inicial, a la discusión sobre la calidad de la propuesta y al imperativo de generar mejores experiencias, para el aprendizaje de nuestros niños y niñas de cero a seis años.

Presentar este estudio ${ }^{1}$, tiene también el propósito de compartir y remirar respecto de cómo hemos venido concibiendo y construyendo el currículum del Jardín Infantil. Creemos que dada la experiencia educativa formal que en general hemos vivido en nuestro país, en gran parte de los establecimientos educacionales, las tareas pedagógicas,

${ }^{1}$ Este artículo monográfico se extrae de la investigación realizada por la autora en el marco del Doctorado en Mediación Pedagógica "Establecimiento de patrones y autoorganización, dos pilares fundamentales para el juego y el aprendizaje" y que también fue aporte a la investigación FONDECYT desarrollada entre los años 2011 y 2015, "Asombros educativos infantiles y propensión a Aprender". 
incluyendo los niveles iníciales pone ya, un fuerte énfasis en mecanismos que permitan controlar la gestión, por lo general más administrativa que pedagógica, para medir los comportamientos humanos, por medio de fórmulas matemáticas predictivas, y no en los procesos cotidianos de interacción e interrelación humana, que dan mayor cuenta de los reales aprendizajes.

\section{Desarrollo}

Es dable considerar que uno de los desencuentros epistemológicos que existe entre los adultos y niños, es que unos buscan las mejores formas y nuevas estrategias para "hacerle frente" a la vida, y los otros transitan y fluyen dinámicamente en ese mismo espacio y tiempo en una dimensión histórica - experiencial, constructiva y placentera. Lo primero, porque la epistemología adulta, por lo general, le da más cabida a orientaciones teóricas que nos hablan de formas de aprender y conocer; así nos hemos ido especializando en “aprender" episódicamente, porque tenemos que ser capaces de describir y definir todo, en cambio los niños, nos muestra que el conocimiento puede transitar desde la inconsciencia a la conciencia, por medio de un estado o flujo creativo que ocurre por la desaparición de la autoconciencia y que permite incursionar en lo ausente, en lo incierto y en lo ignorado.

Por lo anterior, el juego y el jugar al que alude este artículo, es el juego con sentido propio, genuino y espiritual, que por su condición inmaterial, es complejo e indefinible; existe porque suspende la determinabilidad absoluta que lo podría hacer más comprensible.

Los niños y las niñas como los adultos, estamos construyendo permanentemente conocimientos "cotidianos", independientemente del espacio formal, donde no se detiene porque tiene el sentido y funcionalidad que le otorga esa epistemología cotidiana, es decir, es ella la que marca la forma de cómo nos aproximamos a la epistemología y conocimiento “escolar”. Los niños y las niñas cuando ingresan al Jardín Infantil o a la escuela por primera vez, traen consigo su natural propensión a aprender; sus propios conocimientos y un

2 Rodrigo, M.J; Arnay, J. en su libro "La construcción del conocimiento escolar" presentan una clara distinción entre el conocimiento cotidiano y el escolar. Señala que ambos tipos de conocimientos, no se distinguen necesariamente por su contenido, sino muy particularmente por su epistemología constructiva, por el tipo de escenario sociocultural en que se construyen y por sus procesos de construcción. 
escenario de aprendizaje "previo", que van a utilizar para construir el conocimiento escolar, pero tal como señalan Rodrigo y Arnay (1997), lamentablemente al utilizar estos recursos propios dentro del espacio formal, no sólo manifestarán errores en el contenido de sus propias teorías, sino que también en el sentido que le otorguen y en las maneras de utilizarlas, porque esos resultados serán contrastados con lo esperable en un curriculum preescrito y con indicadores construidos para ello. Por supuesto que como en todo orden de cosas, existen escuelas o docentes que buscan las mejores estrategias para educar desde el encuentro de saberes y ese énfasis territorial enriquece la tarea y abre grandes oportunidades para que los niños y niñas se expresen sin inhibiciones, ni inseguridades.

En el mismo sentido, Catalán, J (2010: 44), se pregunta, si mediante la acción de la escuela se espera producir cambios sustantivos en las concepciones que niños y niñas traen, producto de su actividad constructiva y de su interacción social, ¿cuál es la actividad explicativa que ellos y ellas desarrollan cuando recién ingresan al sistema escolar? y ¿de qué manera se incorpora esa actividad explicativa en la acción pedagógica que se lleva a cabo en el aula? Agregamos e estas interrogantes, la situación compleja de, cómo construir conocimiento dentro de la escuela sin que caigamos en el error de tomar en cuenta los conocimientos cotidianos, sólo para atribuirle significados arbitrarios, con el propósito de convertirlo en una herramienta de anclaje del nuevo conocimiento escolar, para instalar como única verdad, lo que se ha prescrito en el currículum de la institución (Op. cit Rodrigo y Arnay).

Parafraseando a Goldberg (2007), nuestra cultura está dominada por la afición a las taxonomías finitas, a las dicotomías marcadas y a las distinciones binarias, sin embargo, la realidad es más a menudo continua y graduada que acotada por fronteras marcadas. El autor nos insta a ver que tanto la sabiduría, como la competencia, se logran por medio de la acumulación de atractores ${ }^{3}$ que permiten el reconocimiento de patrones en situaciones importantes.

\footnotetext{
3 Goldberg (2007)"Un atractor es una red, un grupo de neuronas estrechamente interconectadas que exiben un patrón estable de actividad en ausencia de estimulacion directa desde el exterior. Estos patrones de actividad que se autoperpetúan se denominan "estados atractores". Los estados atractores son posibles porque 
Al respecto Calvo (2008:58) hace una relación entre aprendizaje y establecimiento de relaciones posibles, distinguiendo entre los tiempos y espacios educativos formales cronológicos y los cotidianos o informales históricos, afirmando que el educador inmerso en el tiempo histórico, puede innovar en la medida que comprende que los hechos pueden tener muchas explicaciones y no una sola. En cambio, el profesor no educador que transita por el tiempo cronológico solo será repetidor, pues nunca comprenderá que la educación no es repetición de relaciones prestablecidas, sino creación de relaciones posibles.

Cabe señalar que en las investigaciones realizadas por Goldberg (Op. cit 2007:175177), las personas con una baja capacidad para razonar mantenian o perdían conocimientos generales y vocabulario, mientras que las personas con alta capacidad para razonar continuaban aumentando el conocimiento y el vocabulario con la edad, incluso hasta los 80 años, es decir que el conocimiento tácito se adquiere informalmente y es precisamente aquello lo que no experimenta ningún declive apreciable con la edad.

Los niños y las niñas, aprovechando su inmensa provisión de potenciales capacidades y de sus experiencias vividas, que son entonces, potenciales redes de relaciones, se ven impulsados a aprender inevitablemente; viven los acontecimientos que les permite absorber el ambiente y armonizar con él. Esta relación que establecen con el medio es captada a través de todos los sentidos y por lo general no es consciente en el momento; cuando llega a serlo, ocurre por medio de procesos metacognitivos que transforman la experiencia en aprendizaje. Taleb, Nassim (2009: 11- 31) ha planteado, que no es extraño ver que un suceso se produce precisamente porque no se esperaba que fuera a ocurrir y que por lo mismo lo que creemos saber, estratégicamente, sea completamente intrascendente, porque la incapacidad de predecir las rarezas implica la incapacidad de predecir el curso de la historia.

\section{Metodología}

las conexiones entre las neuronas dentro del atrator son tan fuertes que la activación de cualquier subconjunto de neuronas es suficiente para mantener en marcha a todo el patrón. El atractor se activa íntegramente, como un conjunto, cada vez que se activa cualquiera de sus componentes". 
Por la complejidad de esta investigación eminentemente cualitativa, se utilizó un modelo de análisis descriptivo, sustentado en la teoría fundamentada, que posee una serie de estrategias metodológicas para realizar el estudio de la información. Por medio de este modelo se fueron reconstruyendo inductivamente categorías generales a partir de elementos particulares o códigos semánticos, que fueron resumiendo en un concepto los elementos esenciales de las situaciones registradas, en este caso experiencias lúdicas, interacciones, diálogos u otras manifestaciones propias de los niños y niñas, sujetos de la investigación. Al finalizar esta etapa se obtuvo una descripción bastante exhaustiva de los contenidos de los registros que fueron mostrando la existencia de patrones subyacentes a las interacciones lúdicas, develando progresivamente en ellas, la existencia de atractores que al parecer son los que permiten su vigencia. La delimitación de la teoría emergente; permitió ir descubriendo uniformidades en el grupo original de categorías, que por su definición conceptual y operacional, finalmente se generó el texto que dio sentido a este estudio.

\section{Conclusión}

A tiempo de concluir, diremos que en el contexto de los juegos infantiles, generalmente existe la tendencia a la transgresión, al caos, a los saltos contextuales y a las secuencias accionales indeterminadas que dependen de las relaciones analógicas que los niños y las niñas generan al entrar en relación con el entorno, no obstante aquello, los juegos mantienen una estructura y su vigencia. Es ahí, en ese espacio subyacente que encontramos lo que podríamos llamar atractores del sistema lúdico.

Como resultado de esta investigación, encontramos algunos como:

\section{- Establecimientos de relaciones analógicas}

Dadas estas relaciones que los niños y niñas establecen con el entorno, van ocurriendo saltos contextuales cada vez que se produce una saturación de la situación o experiencia que están viviendo. La pérdida del estado de bienestar provoca una secuencia accional donde los niños y niñas van probando y buscando nuevas y diversas posibilidades de restablecer un estado más gozoso, por lo general a través de relaciones analógicas de 
diversos tipos: antinómica o de oposición, sinonímica, atributivas, funcionales o pragmáticas, etc.

El razonamiento analógico se percibe como un patrón del aprendizaje, a través de estos desplazamientos metafóricos los niños y las niñas crean mundos diversos en un mismo espacio y cada mundo lo viven como un acto existencial con el cual se comprometen; desarrollan su capacidad creativa para comunicar, representar y expresar la realidad a partir de la elaboración original que hacen desde sus sentimientos, ideas, experiencias y sensibilidad. Durante la investigación, vimos que la representación mental que realizan los párvulos de aquellos elementos y situaciones que evocan ante una nueva propuesta lúdica, es evidente y recurrente. En todo momento los niños y las niñas van creando realidades que surgen de algo que ven, escuchan o tocan. Si tienen esta posibilidad, la exploración les agudiza los sentidos, les permite conocer y aprender activamente el entorno, y proporciona una visión futura de diálogo entre los distintos contextos educativos, cotidianos y formales. Estas realidades que emergen, nos llevan a explorar las conexiones metafóricas y las relaciones análogas que existen entre el pensamiento de niños y niñas y el mundo, lo que nos permite ver, que cosas aparentemente diferentes, son también iguales.

\section{○ Indeterminación probabilística}

Permite la búsqueda del gozo, de las situaciones casuales, ocasionales y fortuitas. En este caso la funcionalidad del atractor, actúa como defensa ante la rigidez de los sistemas escolares, generando tiempos y espacios propicios para explorar, agudizar los sentidos, conocer y aprender activamente del entorno sin un tiempo ni un espacio predeterminado.

En cada uno de los momentos descritos en los registros de campo, se devela este atractor que apunta por lo general a la transgresión de lo establecido, desde cambiar los nombres, decir no, cuando es si, no obedecer, cambiar el final a las canciones, etc. hasta situaciones violentas y agresivas para romper con el poder del adulto en la sala o con la rutina que los aburre.

\section{○ Conductas consensuadas}


Esta teoría, revierte los planteamientos clásicos y lineales de la comunicación. Maturana (2005) señala que toda la vida humana ocurre en conversaciones cuyo fluir es a través del lenguajeo y el emocionamiento, dos elementos fundamentales que surgen con fuerza en esta investigación y que podrían dar sustento a otra característica de funcionalidad de los atractores. La complicidad, la sensibilidad para captar las emociones, la prosodia y la intencionalidad de las personas con quienes los niños y niñas comparten en el Jardín Infantil, es muy potente. Los juegos se arman y desarman por lecturas sutiles que los niños y las niñas hacen de los demás. En este sentido el atractor se devela como una herramienta para conseguir el bienestar, por medio del lenguaje y la emoción.

\section{Ejemplo}

\begin{tabular}{|c|c|c|c|}
\hline Párvulo & Considerando que.. & & \\
\hline $\begin{array}{l}\text { 1.-Samuel se pone de } \\
\text { pie, y se acerca a } \\
\text { Yeison, mira su mesa } \\
\text { y busca la goma, } \\
\text { Yeison la esconde y se } \\
\text { ríe. }\end{array}$ & $\begin{array}{l}\text { Esta acción que podría } \\
\text { haber terminado con } \\
\text { Samuel obteniendo la } \\
\text { goma, se transforma en } \\
\text { una acción lúdica } \\
\text { (gozosa) }\end{array}$ & $\begin{array}{lr}\text { Cuando } & \text { Yeison } \\
\text { incorpora un elemento } \\
\text { que transforma el } \\
\text { contexto, genera una } \\
\text { secuencia } \\
\text { lúdica. accional }\end{array}$ & \\
\hline $\begin{array}{l}\text { 2.-Samuel vuelve a su } \\
\text { asiento, mira a } \\
\text { Martina, ella pone su } \\
\text { estuche tapando su } \\
\text { dibujo. }\end{array}$ & $\begin{array}{l}\text { Samuel interpreta la } \\
\text { reacción de Martina y el } \\
\text { dice ¡estoy mirando! } \\
\text { Martina se ríe. }\end{array}$ & $\begin{array}{l}\text { Luego Yeison también } \\
\text { tapa su dibujo con el } \\
\text { estuche. } \\
\text { Samuel lo mira y le } \\
\text { dice: ¡Yeison igual te } \\
\text { veo! Yeison se ríe. }\end{array}$ & $\begin{array}{l}\text { La reacción de tapar el } \\
\text { dibujo y la sonrisa de } \\
\text { Martina y Yeison } \\
\text { contribuyen a esta } \\
\text { secuencia accional gozosa } \\
\text { y retroalimentan el juego } \\
\text { propuesto por Samuel. }\end{array}$ \\
\hline $\begin{array}{l}\text { 3.-Samuel: } \\
\text { ¡Yeison desapareció } \\
\text { tu lápiz, porque yo lo } \\
\text { tengo! le muestra un } \\
\text { lápiz. } \\
\text { Yeison, mira su mesa } \\
\text { y no encuentra su } \\
\text { lápiz. Yeison lo mira } \\
\text { y le sonríe }(. . .)\end{array}$ & $\begin{array}{l}\text { La acción realizada por } \\
\text { Samuel es compartida } \\
\text { por Yeison }\end{array}$ & $\begin{array}{l}\text { La mirada y la sonrisa } \\
\text { contribuyen a esta } \\
\text { secuencia accional que } \\
\text { se transforma en un } \\
\text { momento de juego, } \\
\text { dentro del espacio } \\
\text { formal del aula. }\end{array}$ & $\begin{array}{l}\text { En la situación descrita en } \\
\text { la primera columna, } \\
\text { encontramos pistas claras } \\
\text { sobre el conocimiento } \\
\text { cotidiano de los tres } \\
\text { párvulos, quienes son } \\
\text { capaces de comunicarse, a } \\
\text { través del lenguaje no } \\
\text { verbal, usan e interpretan } \\
\text { códigos lingüísticos, } \\
\text { estableciendo una relación } \\
\text { analógica atributiva. }\end{array}$ \\
\hline
\end{tabular}

Esta es una muestra de cómo los niños y niñas van construyendo un juego con elementos tan sutiles, que a ojos de un adulto que no está atento a estas interacciones, pasan 
inadvertidas, por lo mismo no comprende el juego y lo interpreta de manera episódica y errónea.

Para estos párvulos bastó una mínima acción para modificar el contexto formal del aula, volviéndolo lúdico y gozoso; se fue retroalimentando con una secuencia de acciones lo que terminó por configurar un juego compartido, significativo y que sin duda al analizarlo de manera más fina, encontraremos que usan perfectamente la experimentación rigurosa y exigente, el razonamiento lógico y la contrastación, tal como lo hace cualquier científico en su tarea, sobrepasando con creces cualquier aprendizaje formal promovido en el jardín infantil. Creemos importante atender a la propensión a aprender, de los seres humanos y en particular en sus primeros años de vida, pues esta fluye a través de procesos autoorganizativos, siguiendo patrones simples y, a la vez, complejos.

\section{Referencias bibliográficas}

Arnay, J; Rodrigo, M. J. (1997). La Construcción del Conocimiento Escolar. Barcelona: PAIDOS.

Calvo, C. (2008). Del mapa escolar al territorio educativo. Disoñando la escuela desde la educación. La Serena: Nueva Mirada Ediciones, $3^{\text {a }}$ edición.

Calvo, C y otros (2011-2014). Asombros educativos infantiles y propensión a aprender. Investigación Fondecyt N¹110577

Catalán, J. (2010). Teorías Subjetivas. Aspectos teóricos y prácticos. La Serena: Editorial Universidad de La Serena.

Goldgber, E (2006) La paradoja de la sabiduría. Barcelona. Drakontos

Maturana, H.(2005). Emociones y lenguaje en educación y política. Santiago de Chile: Ed. Hachette/Comuicacion

Prigogine, L. (1997). El fin de las certidumbres. Santiago de Chile: Ed. Andrés Bello.

Taleb, N. (2009). El cisne negro. PAIDOS

Tolle, E. (2003): El silencio habla. Madrid: Ediciones GAIA. 
Wolff, M. (1994). La cotidianeidad. Madrid: Ediciones Cátedra.

Zohar, D. (1996). El yo cuántico. Naturaleza humana y conciencia definida por la física moderna. Ciudad de México: Publimex.

Educación y conocimiento: eje de la transformación productiva con equidad. (1992). Santiago de Chile: CEPAL-UNESCO 